\title{
PRÁTICAS ESTRATÉGICAS DE INTERNACIONALIZAÇÃO DE PROGRAMAS DE PÓS- GRADUAÇÃO: ESTUDO DE CASO EM UMA UNIVERSIDADE PÚBLICA DO SUL DO BRASIL
}

\author{
Thayse Kiatkoski Neves ${ }^{1}$, Rosalia Aldraci Barbosa Lavarda, Cibele Barsalini Martins
} Universidade Federal de Santa Catarina - UFSC, Santa Catarina, (Brasil)

\section{DETALHES DO ARTIGO}

\section{Histórico do Artigo:}

Recebido em: 04 de julho de 2018

Aceito: 19 de dezembro de 2018

Disponível online: 26 de março de 2019

Sistema de revisão "Double blind review"

Editor Científico

Ilan Avrichir

\section{Palavras-chaves:}

Estratégia como prática social

Internacionalização

Pós-graduação

\section{RESUMO}

A perspectiva da estratégia como prática social surgiu como uma das alternativas às pesquisas tradicionais na área de estratégia organizacional, passando a explicar a estratégia como algo que as pessoas fazem no seu dia a dia e não como uma propriedade das organizações. A internacionalização da pós-graduação brasileira se constitui em uma das principais metas do Sistema Nacional de Pós-Graduação. Nesse sentido, a perspectiva da estratégia como prática social pode ser utilizada para compreender as microdinâmicas relacionadas às estratégias de internacionalização dos programas de pós-graduação, como são efetivamente implementadas as ações e quem são os atores responsáveis por tais implementações. A partir da análise de programas stricto sensu considerados de excelência, segundo os critérios da CAPES, este estudo buscou compreender como se desenvolvem na prática cotidiana as estratégias de internacionalização de programas de pós-graduação stricto sensu de uma universidade pública federal que obtiveram nota máxima na Avaliação Trienal 2013 da CAPES. Adotou-se a metodologia qualitativa, a partir de um estudo de caso conduzido entre programas de uma universidade pública do sul do Brasil. Os resultados evidenciaram o papel de destaque assumido pelos docentes no processo de internacionalização dos programas. Também pode-se verificar que a estratégia acontece rotineiramente a partir do desenrolar das ações cotidianas dos programas abrangidos pelo estudo.

(C) 2018 Internext | ESPM. Todos os direitos reservados!

\section{INTRODUÇÃO}

Em 1996, com a publicação do artigo Strategy as Practice por Richard Whittington, começou a se delinear uma nova perspectiva para se estudar a gestão estratégica e as tomadas de decisões dentro das organizações: a abordagem da "estratégia como prática" (Whittington, 1996). No entanto, é a partir da virada do século que a abordagem da estratégia como prática social passou a ganhar mais espaço nos estudos organizacionais (Johnson, Melin \& Whittington, 2003, Jarzabkowski, 2004; Whittington, 2006; Jarzabkowski, Balogun \& Seidl, 2007).

Considerando as contribuições advindas de áreas como a sociologia e a filosofia, a estratégia como prática compreende a estratégia como uma construção social, ou seja, como algo que as pessoas fazem no seu dia a dia (Whittington, 2006; Jarzabkowski et al., 2007). Dessa forma, afasta-se a visão clássica que trata a estratégia como uma propriedade das organizações (Golsorkhi, Rouleau, Seidl \& Vaara, 2015)

$\mathrm{Na}$ tentativa de retirar o foco sobre os efeitos das estratégias no desempenho organizacional, a estratégia como prática social passa a analisar mais profundamente o que ocorre na dinâmica de formulação, planejamento e implementação das estratégias organizacionais (Golsorkhi et al., 2015). Nessa abordagem, a estratégia surge a partir da interconexão de três elementos-chave: as práticas (practices), as práxis (pratice) e os praticantes (practioners) (Whittington, 2006).

As práticas envolvem as rotinas e atividades desenvolvidas na organização, as quais se desdobram em ações (práxis) que são executadas por todos aqueles atores envolvidos com a organização (praticantes), independentemente do nível de

\footnotetext{
${ }^{1}$ Contato do autor - E-mail: thayse.neves@ufsc.br
} 
atuação. Membros da alta administração, gerentes de nível médio, funcionários do nível operacional e até mesmo consultores externos são analisados a partir de sua relação no processo decisório estratégico das organizações (Jarzabkowski, 2005; Whittington, 2006; Jarzabkowski et al., 2007; Jarzabkowski \& Spee, 2009).

A dimensão internacional é intrínseca às universidades. Desde sua criação, as universidades são consideradas instituições globais e são afetadas por situações e circunstâncias que vão além de seus campi e das fronteiras nacionais (Altbach, 2006). No entanto, é a partir da década de 1990 que a internacionalização da educação superior ganhou mais destaque e relevância no panorama mundial (Altbach, Reisberg \& Rumbley, 2009; Knight, 2004; Morosini, 2006a, 2006b; Stallivieri, 2017a).

No contexto da educação superior brasileira, a pós-graduação adquire papel central no processo de internacionalização, devido à sua conexão com a pesquisa, que se encontra no núcleo desse processo (Laus \& Morosini, 2005; Lima \& Contel, 2009).

O Plano Nacional de Pós-Graduação (PNPG) 20112020, desenvolvido pela Coordenação de Aperfeiçoamento de Pessoal de Nível Superior (CAPES), imputa a busca pela internacionalização como uma das principais metas do Sistema Nacional de Pós-Graduação e destaca que uma das formas de se buscar a excelência e a aquisição de novos conhecimentos se dá pela interação mais intensa entre instituições brasileiras e internacionais. Segundo o PNPG 2011-2020, a internacionalização, além de promover o crescimento da ciência, aumenta o protagonismo do país no cenário internacional (CAPES, 2010). Desde a modificação dos critérios de avaliação da pós-graduação stricto sensu inserida pela CAPES em 1998, a internacionalização tem sido, inclusive, um dos critérios mais relevantes adotados para aferição de qualidade e atribuição dos conceitos de excelência acadêmica aos programas de pósgraduação no país (Lima \& Contel, 2011; Morosini \& Do Nascimento, 2017).

Ratificando a histórica relevância da pósgraduação no processo de internacionalização da educação superior brasileira, a CAPES instituiu, no final de 2017, o Programa Institucional de Internacionalização (CAPES-PrInt) (CAPES, 2017c). A partir da ampliação e do aprimoramento de ações de internacionalização junto a programas de pósgraduação stricto sensu, o Programa CAPES-PrInt busca incentivar a internacionalização de instituições de ensino superior e institutos de pesquisa.

Estudos como os de Bendelier, Kondakci e Zawacki-Richter (2018) e Kuzhabekova, Hendel e Chapman (2015) evidenciam o aumento das discussões sobre a internacionalização da educação superior, em âmbito internacional, com o passar dos anos e reforçam a importância acadêmica do fenômeno. No contexto nacional, Sampaio e Saes (2014) destacam que as primeiras aparições do termo "internacionalização" associado à educação superior em produções científicas datam de 2002, mas passam a ganhar mais ênfase no contexto acadêmico a partir de 2006. No entanto, se comparada à produção científica internacional, a internacionalização da educação superior como objeto de estudos em dissertações e teses ainda é pequena no Brasil (Morosini \& Do Nascimento, 2017).

Além disso, conforme já apontado por Sanderson (2008), a discussão sobre internacionalização ainda foca principalmente no ponto de vista da instituição, deixando por vezes de lado, por exemplo, as ações individuais de pessoas e unidades específicas das instituições, as quais também impactam no processo de internacionalização institucional (Hudzik, 2011; Knight, 2015; Seeber, Cattaneo, Huisman \& Paleari, 2016; Seeber, Meoli \& Cattaneo, 2018)

Nesse sentido, relacionando a abordagem da estratégia como prática social com os aspectos que envolvem as microdinâmicas relacionadas às estratégias de internacionalização de programas de pós-graduação, o objetivo do presente estudo foi compreender como se desenvolvem na prática cotidiana as estratégias de internacionalização de programas de pós-graduação stricto sensu de uma universidade pública federal que obtiveram nota máxima na Avaliação Trienal 2013 da CAPES.

Para consecução do objetivo da pesquisa, adotouse a metodologia qualitativa (Stake, 2003), a partir de um estudo de caso único (Eisenhardt, 1989), realizado em uma universidade pública federal no sul do Brasil. A seleção do caso ocorreu pela busca de um objeto de pesquisa caracterizando programas que tivessem alcançado nota máxima (7) na Avaliação Trienal 2013 da CAPES, tendo em vista que, de acordo com os critérios adotado por aquele órgão, os programas com nota 7 são aqueles avaliados como "muito bom" em todos os quesitos da avaliação e que, dentre outros aspectos, possuem desempenho equivalente ao dos centros internacionais de 
Práticas Estratégicas de Internacionalização de Programas de Pós-Graduação: Estudo de Caso em uma Universidade Pública do Sul do Brasil

excelência em sua área de atuação. As técnicas de coleta de dados adotadas incluíram análise documental e entrevistas semiestruturadas e a análise dos dados foi feita por meio de análise narrativa (Godoi, Bandeira-De-Mello \& Silva, 2006) e pela técnica pattern matching (Trochim, 1989).

O presente trabalho se encontra estruturado em cinco seções, além desta introdução. Na sequência, faz-se uma descrição dos principais conceitos e estudos relacionados à abordagem da estratégia como prática social e ao fenômeno da internacionalização da educação superior. Em seguida são descritos os procedimentos metodológicos adotados na pesquisa e, após, passase à análise dos dados coletados. Conclui-se com as considerações finais do estudo.

\section{ESTRATÉGIA COMO PRÁTICA SOCIAL}

Desde a década de 1960, quando do surgimento do pensamento estratégico (Chandler, 1962; Ansoff, 1965; Andrews, 1971), até o início da década de 1990, predominava nos estudos organizacionais uma concepção de estratégia como um plano racionalmente pensado pela alta gerência, cujo objetivo primordial era lucratividade das organizações (Whittington, 2002; Volberda, 2004).

Embora tal concepção ainda domine os livrostexto de Administração nos dias atuais, a estratégia evoluiu de um conceito mais prescritivo (o que as organizações devem fazer) para modelos mais descritivos, sobre o que as organizações de fato fazem para ser bem-sucedidas, por meio da introdução de uma dimensão humana e social no processo de formulação e implementação da estratégia, dimensão essa ausente na racionalidade proposta pelas perspectivas clássicas (Cunningham \& Harney, 2012).

O contexto de crescente insatisfação e frustração com os modelos normativos e prescritivos contribuiu para o delineamento e o crescimento de uma nova abordagem de compreensão da estratégia organizacional: a perspectiva de estratégia como prática social (Jarzabkowski, 2005; Whittington, 2006; Jarzabkowski \& Spee, 2009).

Em 1996, em seu artigo Strategy as Practice, Richard Whittington propõe que a estratégia seja tratada como uma prática social, ressaltando a necessidade de se mudar o foco das pesquisas para como os praticantes fazem a estratégia e entender o processo constante de se fazer a estratégia em uma organização (strategizing). Nesse sentido, nos últimos vinte anos, estudiosos dessa abordagem passaram a estudar como os praticantes da estratégia agem e interagem nas organizações, suas habilidades, destrezas e desempenhos, não se preocupando em prescrever ações para o alcance do resultado da organização (Whittington, 1996; Canhada \& Rese, 2009; Jarzabkowski \& Spee, 2009; Vaara \& Whittington, 2012; Jarzabkowski, Kaplan, Seidl \& Whittington, 2016; Brandt, Lavarda \& Lozano, 2017).

Nessa perspectiva, o foco se transfere para as atividades cotidianas, socialmente realizadas por meio de ações e interações dos atores envolvidos, não somente as formais, mas as que podem ter consequências significativas para as organizações e para as pessoas (Jarzabkowski, 2005; Canhada \& Rese, 2009; Lavarda, Canet-Giner \& Peris-Bonet, 2010; Silva, Carrieri \& Junquilho, 2011; Junquilho, Almeida \& Leite-Da-Silva, 2012; Zwick, Silva \& Brito, 2014; Cardoso \& Lavarda, 2015; Brandt et al., 2017).

A estratégia começa, portanto, a ser vista não como algo que as organizações têm, mas sim como fruto da ação e interação humana (Jarzabkowski, 2004; Whittington, 2006; Jarzabkowski et al., 2007). Passa-se a levar em consideração não apenas as atividades da alta cúpula das organizações, mas também aspectos micro-organizacionais e a investigação sobre os gerentes de nível médio e outros atores (Johnson et al., 2003; Jarzabkowski et al., 2007; Jarzabkowski \& Spee, 2009).

A partir do entendimento de estratégia como uma prática social, a análise se desloca um nível abaixo para tratar dos processos estratégicos gerais e das atividades daqueles que praticam a estratégia, considerando não mais a performance dos estrategistas, mas sim o modo como estes desempenham seus papéis (Brandt et al., 2017; lasbech \& Lavarda, 2018). Sob essa ótica, a estratégia pode ser definida como uma atividade situada, realizada socialmente, construída por meio de ações, interações e negociações de múltiplos atores que realizam a prática estratégica (Jarzabkowski, 2005; Jarzabkowski et al., 2007; Jarzabkowski \& Spee, 2009). Refere-se a "fazer a estratégia", que consiste na construção do fluxo de atividade por meio das ações e interações de múltiplos atores e as práticas em que estes baseiam (Jarzabkowski et al., 2007).

Segundo Whittington (2006), a estratégia como prática se baseia em três elementos-chave: as 
práticas (practices), a práxis (practice) e os praticantes (practitioners). Apesar de discretos, esses três elementos-chaves se encontram interconectados de forma que não é possível estudar um elemento isoladamente sem recorrer a aspectos dos outros. À interconexão entre esses três elementos dá-se o nome de strategizing, ou seja, "fazer a estratégia" (Jarzabkowski et al., 2007).

As práticas se referem às ferramentas sociais, simbólicas e materiais por meio das quais as estratégias ocorrem, ou seja, correspondem a rotinas de comportamento compartilhadas, incluindo tradições, normas e procedimentos para pensar e agir dentro da organização e, por isso, estão intrinsecamente ligadas ao "fazer".

Em contrapartida, o termo grego práxis se refere à ação em si, ao 'como' as pessoas fazem no presente, ou seja, considera a atividade atualmente desenvolvida, ensejando a ideia do uso reflexivo das práticas conceituadas acima, quando aquilo que é institucionalmente aceito passa a fazer parte da experiência vivida dos atores da organização. Práxis compreende a interligação entre as ações de diferentes indivíduos e grupos dispersos e as instituições sócio, político e economicamente incorporadas dentro da qual os indivíduos agem e para quem contribuem (Jarzabkowski, 2005; Whittington, 2006; Jarzabkowski et al., 2007; Jarzabkowski \& Spee, 2009).

Já os praticantes da estratégia são todos aqueles profissionais que fazem o trabalho de elaboração, modelagem e execução das estratégias, não se restringindo apenas à alta administração das organizações (Whittington, 2006). Cada vez mais estudos sobre a estratégia como prática social indicam que os gerentes de nível médio e os trabalhadores de nível inferior são importantes atores estratégicos das organizações. São eles os motores principais da estratégia, pois criam, moldam e executam a estratégia. Esses praticantes podem ser tanto os "estrategistas de fato", quanto os gerentes que implementam, até os consultores que muitas vezes participam da vida organizacional. Nesse sentido, torna-se importante identificá-los como estrategistas, pesquisando além da alta administração e incorporando funcionários de múltiplos níveis como atores estratégicos (Lavarda et al., 2010).

Jarzabkowski et al. (2016) sustentam que é importante examinar as práticas dentro de seu contexto, prestando atenção a quem está envolvido e como esses atores trabalham. Essa perspectiva prática também enfatiza que os resultados estratégicos dependem da interação entre o "que" (práticas), com "quem" (praticantes) e "como" (práxis) se relacionam as práticas estratégicas, considerando o ambiente e os resultados pretendidos.

\section{A INTERNACIONALIZAÇÃO DA EDUCAÇÃO SUPERIOR}

O entendimento da dimensão internacional na educação superior passou por mudanças ao longo da história. De uma noção estática e fragmentada no final do século $X X$, tal dimensão passou a ser mais fortemente compreendida como um processo abrangente e estratégico, designado como "internacionalização da educação superior" (Knight 2004; Morosini, 2006b; De Wit 2013). No entanto, De Wit (2013) ainda argumenta ser bastante comum encontrar na literatura e nas ações práticas de internacionalização a utilização de termos que enfatizam uma lógica específica do processo, geralmente relacionada ao currículo - estudos internacionais, estudos globais, etc. - ou à mobilidade acadêmica.

Embora a internacionalização da educação superior possa ser compreendida sob diferentes óticas, adquirir significados distintos e ser abordada de diversas maneiras de acordo com cada contexto (Knight, 2004; De Wit, 2013), o discurso dominante não a trata como um processo desejável ou optativo, mas sim como um processo imperativo e sem voltas, em que o engajamento e comprometimento de toda a instituição se torna necessário para o alcance dos objetivos almejados (Hudzik, 2011).

Cabe destacar que a internacionalização não é uma finalidade, mas um processo para se atingir a um objetivo estabelecido, como, por exemplo, elevar a reputação de uma instituição (Hudzik, 2011). Trata-se de um fenômeno carregado de tensões e contradições, cada vez mais abordados nas discussões sobre o tema, tais como a evasão de cérebros (ou brain drain), o produtivismo acadêmico, o isomorfismo institucional, a imposição e homogeneização cultural, a existência de nação hegemônicas (ativas) e hegemonizadas (passivas) no processo (Lima \& Maranhão, 2009; Lima \& Contel, 2011), entre outras questões que situam o processo dentro de uma matriz cultural de poder colonial (Shahjahan, 2016; Stein, 2017; Leal \& Moraes, 2018). No entanto, o discurso dominante sobre a 
Práticas Estratégicas de Internacionalização de Programas de Pós-Graduação: Estudo de Caso em uma Universidade Pública do Sul do Brasil

internacionalização, na maioria das vezes, relaciona o processo a contribuições positivas, atreladas à qualidade, à excelência, à inovação e ao desenvolvimento (Morosini \& Do Nascimento, 2017; Stallivieri, 2017b).

Conforme definido por Knight (2004) e complementado por De Wit e Hunter (2015), a internacionalização da educação superior pode ser compreendida como a integração intencional de uma dimensão internacional, intercultural ou global nos propósitos, funções e ofertas de educação póssecundária, a fim de aumentar a qualidade da educação e da pesquisa para todos os estudantes e funcionários da instituição, e fazer uma contribuição significativa para a sociedade (De Wit \& Hunter, 2015, tradução nossa).

Outro conceito amplamente utilizado para tratar do tema diz respeito ao conceito de internacionalização abrangente (comprehensive internationalization), proposto por Hudzik (2011), segundo o qual a internacionalização é o compromisso de inserir perspectivas internacionais em todas as áreas da instituição de educação superior. Nesse sentido, toda comunidade acadêmica se torna protagonista do processo: gestores, professores, funcionários e alunos.

No intuito de se internacionalizar, as instituições de ensino superior se utilizam de diferentes estratégias nesse processo. Knight (2004) divide tais estratégias em dois eixos: as estratégias acadêmicas, as quais estão relacionadas ao ensino, à aprendizagem, à pesquisa e a outras atividades de cunho acadêmico; e as estratégias organizacionais, que incluem as políticas, os procedimentos, os sistemas e a infraestrutura de apoio que facilitam e são responsáveis pela institucionalização e pela sustentabilidade da dimensão internacional na instituição. Entre as principais atividades ligadas às estratégias acadêmicas, trazidas pela autora, estão os programas de mobilidade acadêmica, a internacionalização do currículo, os programas de professores visitantes, a realização e publicação de pesquisas em colaboração com pesquisadores estrangeiros, entre outras atividades de colaboração acadêmica. Já entre as estratégias organizacionais pode-se citar o desenvolvimento de políticas institucionais voltadas à internacionalização, a alocação de recursos para programas e atividades de internacionalização, o apoio a estudantes de intercâmbio (incoming e outgoing), entre outras atividades que demonstrem o comprometimento institucional para com a internacionalização da instituição.

No Brasil, tanto a educação superior quanto seu processo de internacionalização sempre estiveram fortemente subordinados ao Estado (Laus \& Morisini, 2005; Lima \& Contel, 2009, 2011). A internacionalização da educação superior no Brasil foi, inicialmente, uma tentativa de revolucionar as instituições e mais tarde fortalecer a pós-graduação, sendo que apenas nas últimas décadas, o processo se tornou uma união de forças e ações para tentar trazer um caráter internacional às funções acadêmicas em uma esfera de ação mais ampla (Laus \& Morosini, 2005).

Segundo Lima e Contel (2009, 2011), as primeiras políticas de cooperação internacional no país começaram a se desenvolver a partir das décadas de 1920 e 1930, com a presença de professores visitantes estrangeiros nas universidades brasileiras, a fim de consolidar o projeto acadêmico das instituições emergentes.

Os autores ressaltam que, desde 1930 até os dias atuais, o processo de internacionalização no Brasil passou por diversas modificações, levando em consideração as motivações para se internacionalizar e o contexto político-econômico-social brasileiro em cada período. No entanto, é a partir do final da década de 1990 que o tema da internacionalização da educação superior adquiriu mais força e relevância no cenário nacional, quando a CAPES explicitou a necessidade do estabelecimento de padrões internacionais para a avaliação das atividades da pósgraduação e das pesquisas (Laus, 2012).

Apesar do processo de internacionalização da educação superior brasileira datar da década de 1920, as pesquisas sobre o contexto nacional começaram a surgir no meio acadêmico a partir dos anos 2000, tratando inicialmente questões relativas à mobilidade acadêmica e, mais tarde, incorporando outras discussões, tais como as estratégias de internacionalização utilizadas por instituições brasileiras, os processos de comercialização da educação superior e a internacionalização do currículo (Sampaio \& Saes, 2014).

\section{A Internacionalização da Pós-Graduação Brasileira}

A pós-graduação, caracterizada pela indissociabilidade entre o ensino e a pesquisa e responsável pela produção de conhecimento, 
desempenha papel fundamental no processo de internacionalização da educação superior, tanto no Brasil quanto ao redor do mundo (Laus \& Morosini, 2005; De Wit, 2013; Ramos, 2018)

Considerando a autonomia do pesquisador e a sua busca por relações internacionais para o desenvolvimento do conhecimento, a internacionalização na função acadêmica de pesquisa, vinculada principalmente às atividades de pós-graduação, desenvolve-se de forma mais ágil (Morosini; 2006b) e as instituições que contemplam a pesquisa entre suas atividades principais dispõem de maior potencial de internacionalização que aquelas focadas apenas no ensino (Laus \& Morosini, 2005).

Duarte, Castro, Cruz e Miura (2012) destacam que na esfera da pós-graduação as ações de internacionalização resultam mais de atividades isoladas e individualizadas dos docentes do que de um processo formal dirigido pela instituição, como acontece na esfera da graduação. Nesse sentido, a internacionalização dos programas de pós-graduação caracteriza-se pela relação de dependência da instituição com os seus docentes e suas redes de relacionamento, percepção esta também evidenciada nos estudos de Romani-Dias, Carneiro e Barbosa (2017) e Ramos (2018). A experiência acadêmica internacional do pesquisador, sua inserção em redes de colaboração internacional, suas coautorias internacionais e sua experiência em publicações internacionais são alguns dos fatores que impactam na internacionalização de instituições de ensino superior (Romani-Dias, Carneiro \& Barbosa, 2017).

Marrara (2007) destaca que os objetivos da internacionalização, do ponto de vista da educação, podem ser resumidos a uma hipótese de natureza predominantemente institucional e outra de natureza principalmente acadêmica, na qual se insere a internacionalização da pós-graduação. Nesse sentido, o autor conceitua a internacionalização da pós-graduação brasileira como um processo composto por medidas de cooperação internacional necessárias para que um determinado programa de pós-graduação complemente a capacitação de seus discentes e docentes, com o objetivo de estimular o progresso da ciência e a solução de problemas brasileiros e comuns da humanidade por meio da colaboração e da troca de experiências com agentes estrangeiros. Dentro dessa perspectiva, a internacionalização se desenvolve tanto por meio de atividades discentes, envolvendo mestrandos e doutorandos, quanto docentes, tais como a realização de estágios de pesquisas no exterior, participação em cursos e congressos internacionais, celebração de co-tutelas e co-orientações.

No ano de 2017, um estudo desenvolvido pela CAPES junto aos programas de pós-graduação das instituições de ensino superior brasileiras com o intuito de diagnosticar a atual situação dessas instituições demonstrou que, embora a internacionalização não se configure mais em um processo incipiente, ajustes são necessários para torna-la mais eficiente (CAPES, 2017b). O estudo evidenciou, ainda, a forte tendência nacional à internacionalização passiva, principalmente através da mobilidade de docentes e discentes para o exterior, bem como a dificuldade de internalização do conhecimento obtido no exterior e a carência de planos estratégicos de internacionalização alinhados com as políticas da CAPES para esse tema.

Além disso, os resultados obtidos pela CAPES serviram de base para o desenvolvimento de um novo programa voltado à implementação de estratégias de internacionalização nas universidades brasileiras. O Programa Institucional de Internacionalização - CAPES-PrInt (CAPES, 2017c), lançado no final de 2017, visa à ampliação e ao aprimoramento de ações de internacionalização da pós-graduação, além de objetivar a construção, implementação e consolidação de planos estratégicos de internacionalização. De acordo com suas competências e a partir da definição de áreas prioritárias para a internacionalização, as instituições contempladas pelo programa desenvolverão, além de ações voltadas à mobilidade passiva, ações de internacionalização ativa que prevejam estratégias para a consolidação de parcerias internacionais existentes e a construção de novas parcerias, a consolidação projetos de cooperação para aumento da interação entre a instituição brasileira e grupos de pesquisa no exterior, a atração de discentes estrangeiros e a atração e fixação de pesquisadores estrangeiros no Brasil.

Além do récem-institído Programa CAPES-PrInt, a própria CAPES e outras agências de fomento à pesquisa, como o Conselho Nacional de Desenvolvimento Científico e Tecnológico (CNPq), e as fundações de amparo à pesquisa em nível estadual dispõem de diversos outros programas de fomento a 
Práticas Estratégicas de Internacionalização de Programas de Pós-Graduação: Estudo de Caso em uma Universidade Pública do Sul do Brasil

pesquisadores vinculados a programas de pósgraduação. Tais iniciativas reforçam a centralidade da pós-graduação no processo de internacionalização da educação superior brasileira.

A CAPES é responsável ainda pela avaliação do Sistema Nacional de Pós-Graduação. A avaliação dos programas de pós-graduação, iniciada em 1976 e modificada para sua atual estrutura em 1998, consiste em um instrumento de grande importância à concessão de auxílios, tanto por parte das agências de fomento nacionais, como dos organismos internacionais e, atualmente, é realizada a cada quatro anos (CAPES, 2015). Entre os objetivos da avaliação está a certificação da qualidade da pósgraduação brasileira, que serve como referência para a distribuição de bolsas e recursos para o fomento à pesquisa (CAPES, 2017d). Além disso, os estudos e indicadores produzidos pela avaliação servem também para induzir políticas governamentais de apoio e crescimento da pós-graduação (CAPES, 2017a).

Conforme o desempenho acadêmico no quadriênio, os programas recebem notas que variam de 1 a 7, sendo que a aferição de notas 6 e 7 fica restrita a programas reconhecidos como "de nível internacional", e os programas de nota 7 são aqueles com desempenho claramente destacado dos demais, inclusive dos de nota 6 (CAPES, 2015). Nesse sentido, a internacionalização se constitui em um critério basilar para a avaliação de qualidade em programas de pós-graduação de excelência (Morosini \& Do Nascimento, 2017). Para além da exploração de acordos internacionais, é esperado que esses programas de excelência apresentem nível de qualificação, de produção e de desempenho equivalentes ao dos centros internacionais de excelência na formação de recursos humanos, sendo a avaliação da pós-graduação uma indutora do processo de internacionalização dos programas de pós-graduação (Maccari, Lima \& Riccio, 2009).

\section{METODOLOGIA}

Esta pesquisa se classifica, do ponto de vista da abordagem do problema, como qualitativa. Segundo Minayo (2002), a pesquisa qualitativa responde a questões muito particulares e se preocupa com um nível de realidade que não pode ser quantificado. Do ponto de vista da abordagem dos objetivos, a

\footnotetext{
${ }^{2}$ Cabe ressaltar que até o ano de 2012 a avaliação da
} CAPES era realizada trienalmente. A partir do ano de pesquisa caracteriza-se como descritiva, pois expõe características do fenômeno estudado (Vergara, 2010).

Tendo em vista que o presente artigo desconsidera as contradições e os dilemas associados ao fenômeno da internacionalização da educação superior, entende-se que a pesquisa em questão segue uma perspectiva multiparadigmática entre os paradigmas funcionalista e interpretativo. O paradigma funcionalista procura explicar o que acontece no mundo social na busca de regularidade e relações causais entre seus elementos constituintes; enquanto o paradigma interpretativo mantém a preocupação com a realidade social, comum ao paradigma funcionalista, embora do ponto de vista subjetivista, pois se fundamente na visão de que as pessoas constroem e mantêm simbólica e socialmente suas próprias realidades organizacionais (Morgan, 1980; Goles \& Hirschheim, 2000, p. 259; Godoi et al., 2006).

Adotou-se o método de estudo de caso único, definido como uma investigação empírica que investiga um fenômeno contemporâneo dentro de seu contexto da vida real, com o objetivo de explicálos de forma global e tendo em conta toda a sua complexidade (Eisenhardt, 1989). Nesse sentido, o estudo de caso foi desenvolvido com vistas a responder à seguinte questão de pesquisa: Como se desenvolvem na prática cotidiana as estratégias de internacionalização de programas de pós-graduação stricto sensu de uma universidade pública federal que obtiveram nota máxima na Avaliação Trienal 2013 da CAPES?

A seleção do caso se deu de forma não aleatória, intencional e por acessibilidade (Eisenhardt, 1989). As unidades de análise deste estudo foram os três programas de pós-graduação stricto sensu de uma universidade pública federal avaliados com nota 7 na avaliação trienal ${ }^{2}$ da CAPES publicada em 2013. Os programas que compuseram o estudo foram o Programa de Pós-Graduação em Engenharia Mecânica, o Programa de Pós-Graduação em Farmacologia e o Programa de Pós-Graduação em Química.

A fim de assegurar a qualidade e a confiabilidade da pesquisa e como um dos princípios na investigação de estudo de caso, utilizou-se mais de uma fonte de

2013, a avaliação passou a ser realizada de forma quadrienal. 
evidência (Yin, 2005). Para consecução do objetivo do estudo, foram utilizadas como fontes de coleta de dados: análise documental e entrevistas semiestruturadas com os coordenadores dos programas de pós-graduação (Godoi et al., 2006).

A análise documental consistiu na verificação das informações nos relatórios submetidos pelos programas nos anos de 2013 a 2016 junto à Plataforma Sucupira da CAPES, o que propiciou a identificação de ações de internacionalização adotadas pelos programas no período avaliativo da CAPES, bem como na verificação das Fichas de Avaliação dos programas referentes à Avaliação Trienal 2013, as quais contêm os pareceres das comissões de área em relação à internacionalização dos programas. As entrevistas com os coordenadores dos programas ocorreram na segunda quinzena do mês de junho de 2017 e seguiram um roteiro semiestruturado, no qual se buscou explorar aspectos relativos à formulação e implementação das estratégias de internacionalização dos programas, bem como compreender os atores envolvidos no processo, e tiveram duração média de 40 minutos. As principais questões-guia das entrevistas foram: Como são determinados os objetivos da internacionalização? Como surgem as ações de internacionalização? Quem são os responsáveis pela execução dessas ações? Como a internacionalização é incentivada pelo programa?

A análise de dados foi desenvolvida por meio da análise da narrativa (Godoi et al., 2006), a partir da definição dos elementos constitutivos de análise (Kerlinger, 1979), os quais orientaram tanto a fase de coleta quanto de análise dos dados, e também da utilização da técnica de pattern matching (Trochim, 1989), que consiste na comparação da construção teórica com os dados coletados nas entrevistas e análise dos documentos, na realidade observada. Para operacionalizar a fase de análise de dados, utilizaram-se os elementos constitutivos de análise a partir de Kerlinger (1979), considerando dois tipos de definições: a constitutiva (DC) e a operacional (DO). A partir do construto teórico da pesquisa e do objetivo proposto, foram definidos, então, três elementos constitutivos de análise (EC), conforme Figura 1.

\begin{tabular}{|c|c|c|c|}
\hline Elemento & $\begin{array}{c}\text { Definição Constitutiva } \\
\text { (DC) }\end{array}$ & Definição Operacional (DO) & $\begin{array}{c}\text { Constructo } \\
\text { Teórico } \\
\end{array}$ \\
\hline Práticas & $\begin{array}{l}\text { Rotinas, atividades e ações } \\
\text { cotidianas realizadas dentro da } \\
\text { organização (incluindo } \\
\text { tradiçôes, normas e } \\
\text { procedimentos) }\end{array}$ & $\begin{array}{l}\text { Buscou-se identificar essas práticas de } \\
\text { internacionalização por meio de perguntas } \\
\text { específicas nas entrevistas semiestruturadas e } \\
\text { pela análise documental, quais as atividades } \\
\text { caracterizam a internacionalização }\end{array}$ & \multirow{3}{*}{$\begin{array}{c}\text { Whittington, 2006; } \\
\text { Jarzabkowski et al., } \\
2007\end{array}$} \\
\hline Práxis & $\begin{array}{l}\text { Ações dos indivíduos } \\
\text { (praticantes); como estes } \\
\text { realizam as práticas }\end{array}$ & $\begin{array}{l}\text { Buscou-se analisar as práxis por meio de } \\
\text { perguntas específicas na entrevista } \\
\text { semiestruturada e análise de documentos, } \\
\text { buscando identificar, por exemplo, como os } \\
\text { indivíduos envolvidos com os programas } \\
\text { viabilizavam a execução das atividades voltadas } \\
\text { à internacionalização no dia a dia da Instituição }\end{array}$ & \\
\hline Praticantes & $\begin{array}{l}\text { Atores da estratégia, os } \\
\text { estrategistas que executam as } \\
\text { atividades, podendo ser } \\
\text { pertencentes a qualquer nível } \\
\text { hierárquico da organização ou } \\
\text { até mesmo externos a ela } \\
\end{array}$ & $\begin{array}{l}\text { Buscou-se analisar, por meio das entrevistas e } \\
\text { dos documentos verificados os atores internos e } \\
\text { externos à universidade que possuem algum } \\
\text { grau de influência nas ações de } \\
\text { internacionalização desenvolvidas nos âmbitos } \\
\text { dos programas }\end{array}$ & \\
\hline
\end{tabular}

Figura 1: Elementos constitutivos de análise

Fonte. Elaboração própria

Diante do exposto, passa-se à análise dos resultados, articulando os elementos constitutivos - práticas, práxis e praticantes - aos dados coletados na pesquisa.

\section{Análise dos Resultados}

A análise dos resultados está composta pela caracterização da organização objeto de estudo e apresentação dos elementos constitutivos de análise, a partir dos dados coletados nas entrevistas e nos documentos de forma integrada. Caracterização da Instituição e dos Programas de Pós-
Graduação Analisados

A universidade estudada é uma universidade pública, multicampi, fundada em 1960 e que conta 
Práticas Estratégicas de Internacionalização de Programas de Pós-Graduação: Estudo de Caso em uma Universidade Pública do Sul do Brasil

com uma comunidade constituída por cerca de 50 mil pessoas, entre docentes, técnicos-administrativos e estudantes.

Possui 67 programas de pós-graduação acadêmicos em funcionamento, compostos por 65 cursos de mestrado e 56 cursos de doutorado (UFSC, 2017).

O Programa de Pós-Graduação em Engenharia Mecânica foi o primeiro programa de pós-graduação da Universidade, tendo implantado o curso de mestrado no ano de 1969 e, posteriormente, em 1981, o curso de doutorado. Nas avaliações trienais realizadas pela CAPES referentes aos anos de 2010 e 2013, o programa foi avaliado com conceito 7, tendo sido anteriormente avaliado com nota 6 por cinco triênios consecutivos.

De acordo com parecer exarado pela comissão de área na ficha de avaliação do programa referente à avaliação trienal 2013 da CAPES (CAPES, 2014), a internacionalização do programa se evidencia pelos diversos convênios de cooperação firmados com centros de excelência no exterior, os quais promovem o intercâmbio de docentes e alunos entre as instituições. Além disso, a participação dos docentes em eventos internacionais e suas importantes colocações em associações, conselhos e comissões nacionais e internacionais destacam sua internacionalização.

O Programa de Pós-Graduação em Farmacologia, por sua vez, iniciou suas atividades em 1991 com o curso de mestrado e em 1997 implantou também o curso de doutorado.

O programa recebeu nota 7 nas avaliações trienais de 2010 e 2013. Segundo a comissão responsável pela avaliação trienal 2013, o programa atende a todos os critérios de excelência, liderança e internacionalização (CAPES, 2013a).

O Programa de Pós-Graduação em Química foi criado em 1971, com a implantação do curso de mestrado, e em 1988 implantou o curso de doutorado. O programa possui um foco acadêmico e atua na formação de pessoas qualificadas para o exercício da docência em instituições de ensino superior e para o desenvolvimento da ciência e da tecnologia de forma interdisciplinar.

A comissão responsável pela avaliação trienal 2013 da CAPES considerou o programa apropriado ao conceito 7 por apresentar indicadores compatíveis aos de centros de excelência no exterior (CAPES, 2013b).

\section{Práticas}

Partindo-se da análise dos relatórios de dados submetidos pelos programas na Plataforma Sucupira da CAPES e das informações constantes em seus sítios eletrônicos, buscou-se, incialmente, evidenciar aspectos relacionados à inserção internacional dos programas que estivessem explicitados em documentos oficiais, uma vez que nenhum dos programas analisados dispõe de um planejamento estratégico próprio.

Apesar de não contemplarem estratégias deliberadas de internacionalização, todos os programas possuem em seus objetivos geral e/ou específicos aspectos em algum grau relacionados à inserção internacional, seja do próprio programa ou de seus discentes ou egressos.

As práticas, de acordo com a perspectiva da estratégia como prática social, envolvem as rotinas, atividades e ações cotidianas realizadas no dia a dia das organizações, as quais incluem tradições, normas e procedimentos adotados (Whittington, 2006; Jarzabkowski et al., 2007). Nesse sentido, as práticas de internacionalização se configuram nas atividades e rotinas adotadas pelos programas com vistas à sua inserção internacional, ao alcance de desempenho equivalente ao de centros internacionais de excelência na área, conforme estabelecido pela CAPES, e ao seu reconhecimento no âmbito internacional.

As práticas de internacionalização utilizadas pelos programas estão, primordialmente, voltadas ao estabelecimento de cooperações internacionais com programas, núcleos, grupo de pesquisa ou laboratórios de universidades estrangeiras. Ressaltase, no entanto, que nem sempre essas parcerias são institucionalmente formalizadas por meio de termos ou acordos de cooperação entre os entes envolvidos, respaldando-se, por vezes, na informalidade dos contatos entre os docentes das diferentes instituições.

A autonomia e a informalidade no processo de internacionalização no âmbito da pós-graduação evidenciam a existência de um paradoxo, conforme já apontado por Duarte et al. (2012).

Se, por um lado, garantem a independência e o poder do docente em relação à instituição, por outro, 
dificultam o controle do processo de internacionalização pela própria instituição.

Entre as práticas de internacionalização identificadas estão (i) a realização de intercâmbios por docentes (pós-doutoramento) e discentes dos programas (principalmente relacionados à realização de doutorados-sanduíche); (ii) a realização de projetos em cooperação com pesquisadores de universidades estrangeiras, os quais resultam muitas vezes em (iii) publicações conjuntas em periódicos indexados de alta relevância internacional; (iv) a participação de docentes na organização de conferências e seminários internacionais, bem como (v) a apresentação de trabalhos por docentes e discentes em eventos internacionais de alta relevância na área de atuação do programa; e, (vi) a celebração de cotutelas de doutorado. Segundo um dos entrevistados:

A internacionalização do programa, ela tem se dado pelo fato de que muitos dos nossos docentes têm trabalhos em colaboração com colegas de universidades no exterior [...].

Quando você faz um trabalho, que é um trabalho bastante sério, e isso é demonstrado pelo fato de que as nossas pesquisas são publicadas em revistas de muita qualidade, de um bom impacto aí fora, fica muito mais fácil de a gente ter as portas abertas para conseguir esses diálogos. E aí, a gente consegue, dessa forma, "barganhar" apoios aí fora, para que, por exemplo, os nossos alunos possam ir fazer parte de suas pesquisas de doutorado ou sair para fazer pós-doutorado (Entrevistado 2).

Em menor escala, e ainda não utilizada por todos os programas, está (vii) a oferta de disciplinas em idioma estrangeiro (inglês) e (viii) a vinda de docentes de universidades estrangeiras para ministrar minicursos, seminários ou participar em projetos de pesquisa, mencionada como prática já consolidada por um dos programas analisados.

A partir dos relatos dos coordenadores e do observado durante a pesquisa, as práticas de internacionalização atualmente adotadas pelos programas nasceram das ações dos docentes dos cursos, desde o início da criação dos programas, e foram se consolidando como práticas institucionalizadas ao longo do tempo, o que as tornam atividades estratégicas de internacionalização, corroborando com os achados de Duarte et al. (2012). Segundo Johnson, Melin e
Whittington (2003), uma atividade passa a ser estratégica na medida em que traz consequências para os resultados estratégicos da organização, para a sua sobrevivência e para a sua vantagem competitiva, mesmo quando tais consequências não fazem parte de uma estratégia formal e pretendida.

Esta participação e iniciativa dos docentes também se alinham às propostas de Nketia (2016) que destaca o envolvimento de todos os participantes no strategizing, caracterizando o que denomina de open strategy ou estratégia aberta.

\section{Práxis}

As práxis estão relacionadas ao modo como as práticas identificadas são de fato implementadas (Whittington, 2006), a interligação entre as ações de diferentes indivíduos e grupos dispersos e as instituições sócio, político e economicamente incorporadas dentro da qual os indivíduos agem e para quem contribuem.

Nos programas analisados, a práxis está atrelada às ações dos docentes dos programas, os quais são os principais responsáveis tanto pela institucionalização das práticas estratégicas, quanto pela execução das ações que levam à realização dessas atividades. De acordo com o coordenador de um dos programas:

A cultura de internacionalização do programa remete à década de 1970. [...] enviou os professores [...] para realizar doutorados no exterior. A partir disso, os professores começaram a criar redes de relacionamento e parcerias com instituições e professores de outros países e, quando retornaram ao país, essas parcerias continuaram a ser exploradas (Entrevista 3).

Por meio das redes de contatos estabelecidas pelos docentes com pesquisadores e/ou instituições estrangeiras, as práticas de internacionalização são executadas e impactam tanto na avaliação do programa junto ao sistema nacional de pósgraduação brasileiro, bem como alavancam seu reconhecimento em âmbito internacional, conforme relatou um dos coordenadores:

A nossa internacionalização aqui ela está muito baseada em relações pessoais de docentes com gente de fora do Brasil; em projetos em colaboração, basicamente isso. Alguns deles derivados de seus pós-doutorados, outros se conheceram em congressos e descobriram que tinham interesses em comum e estabelecem parcerias (Entrevista 1). 
Práticas Estratégicas de Internacionalização de Programas de Pós-Graduação: Estudo de Caso em uma Universidade Pública do Sul do Brasil

Percebe-se, a partir das entrevistas, que os docentes dispõem de autonomia no que tange as ações que visam ao fortalecimento dos laços estabelecidos entre seus laboratórios, núcleos e grupos de pesquisas com instituições e pesquisadores estrangeiros, não sendo percebida uma influência direta da coordenação dos programas, ou até mesmo de hierarquias superiores da Universidade, nas tomadas de decisão relativas às suas atividades. Nesse sentido, a estrutura da coordenação do programa, formada pelo coordenador e o apoio técnico, assume um papel de facilitador do processo de internacionalização, funcionando como uma ponte entre as práticas e as ações dos docentes por meio da viabilização de recursos e ferramentas necessárias para a execução das atividades dos próprios docentes. Entre os papeis desempenhados pela coordenação dos programas está, por exemplo, o provimento de auxílios financeiros tanto para a ida de docentes e discentes ao exterior, quanto para a vinda de pesquisadores estrangeiros ao programa, assim como a tramitação de processos internos visando à formalização de acordos, entre outros.

A partir dos relatos dos coordenadores, percebese que é atribuída à internacionalização um caráter orgânico, ou seja, ela se desenvolve natural e fortemente por meio dos núcleos de pesquisas, grupos e laboratórios. Assim, a importância e a influência da ação do docente no alcance e fortalecimento da inserção internacional dos programas são evidenciadas.

Portanto, o praticante da estratégia de internacionalização não pode ser caracterizado como um único estrategista e sim como todos os participantes do processo, corroborando às pesquisas de Whittington (2006) e enfatizando que o "jeito" que cada um encontra para solucionar uma situação específica (episódio de práxis) caracteriza sua práxis como algo que é institucionalmente aceito e que passa a fazer parte da experiência vivida na organização.

\section{Praticantes}

Os praticantes correspondem aos atores organizacionais responsáveis pela execução das atividades (Whittington, 2006; Jarzabkowski et al., 2007). No âmbito da internacionalização dos programas de pós-graduação, os atores organizacionais correspondem a todos aqueles que impactam no processo, sendo estes internos ou externos à instituição e aos programas.

Mesmo que, formalmente, os coordenadores, docentes, discentes e o apoio técnico (servidores técnico-administrativos) não possam ser designados como estrategistas dentro de uma perspectiva clássica da estratégia, a ação dessas pessoas, mesmo que não intencionais, são significativas para a sobrevivência e alcance dos objetivos propostos, ou seja, para o desenvolvimento e a manutenção dos patamares de internacionalização atingidos pelos programas, podendo, assim, serem caracterizados como practitioners na perspectiva da estratégia como prática social (Jarzabkowski et al., 2007).

Conforme já evidenciado, os principais responsáveis pela execução das práticas que culminam com a inserção internacional dos programas de pós-graduação estudados são os docentes.

Por meio de suas ações diárias, de suas redes de relacionamento, ou seja, pelas suas práxis, estabelecem cooperações internacionais, viabilizam a participação de seus orientandos em programas de doutorado-sanduíche, executam projetos de pesquisa em parceria com pesquisadores estrangeiros e publicam artigos em periódicos de alto impacto internacional, o que evidencia seu papel catalisador como praticantes no processo de internacionalização.

Tais achados vão ao encontro dos resultados apresentados por Duarte et al. (2012, p. 361), que destacam que "na esfera da pós-graduação, as ações de internacionalização resultam menos de procedimentos institucionalizados, e mais de atividades isoladas e individualizadas" e ressaltam a posição de poder do docente em relação à própria instituição, devido à dependência na manutenção de suas redes de relacionamento, principais impulsionadoras da internacionalização.

Nota-se, no entanto, que apesar do peso atribuído aos docentes na consolidação da internacionalização dos programas, os discentes, também na qualidade de pesquisadores (Romani-Dias, Carneiro \& Barbosa, 2017), influenciam esse processo, sendo responsáveis pela manutenção das redes de relacionamento inicialmente estabelecidas e pela ampliação dessas redes, por meio do estabelecimento de contatos com pesquisadores estrangeiros durante sua vivência no exterior. 
A coordenação dos programas assume um papel secundário, mas igualmente importante nesse processo, por permitir a implementação das ações pretendidas pelos docentes. Do mesmo modo está o papel exercido pelas outras unidades vinculadas à administração superior da universidade, como próreitorias e secretarias relacionadas à pós-graduação e às relações internacionais.

Não se pode minimizar o papel estrategista exercido por atores externos às universidades nesse processo, os quais também impactam e influenciam as estratégias dos programas (Jarzabkowski et al., 2007), como é o caso da CAPES, CNPq e das agências estaduais de fomento à pesquisa.

A CAPES não apenas é responsável pela concessão de auxílios e bolsas a alunos e professores, como é o caso, por exemplo, do Programa Professor Visitante do Exterior (PVE), que viabiliza a vinda de professores estrangeiros, e do Programa de Doutorado Sanduíche no Exterior (PDSE), que concede bolsa a doutorandos brasileiros, como também é a principal responsável pela indução de políticas de internacionalização da pós-graduação brasileira.

Assim, considerando a questão de pesquisa previamente estabelecida: como se desenvolvem na prática cotidiana as estratégias de internacionalização de programas de pós-graduação stricto sensu de uma universidade pública federal que obtiveram nota máxima na Avaliação Trienal 2013 da CAPES? Entende-se, a partir das entrevistas e dos documentos analisados, que as estratégias de internacionalização dos programas de pós-graduação stricto sensu que obtiveram nota máxima da Avaliação Trienal 2013 da CAPES, no caso da universidade pública federal estudada, ocorrem de forma articulada fortemente pelas ações dos docentes via redes de contato, atuação nos grupos e núcleos de pesquisa, bem como, pela participação em congressos, publicações e outros eventos internacionais.

\section{CONSIDERAÇÕES FINAIS}

O objetivo deste estudo foi compreender como se desenvolvem na prática cotidiana as estratégias de internacionalização de programas de pós-graduação stricto sensu de uma universidade pública federal que obtiveram nota máxima na Avaliação Trienal 2013 da CAPES. Entende-se que, a partir das evidências apresentadas nos elementos constitutivos de análise, foi possível alcançar o objetivo proposto.
Ao analisar as práticas de internacionalização dos programas de pós-graduação, como estas são realizadas (práxis) e quem as realizam (praticantes) (Whittington, 2006), pode-se perceber que essas práticas se delineiam e se estabelecem, principalmente, a partir das ações dos docentes vinculados aos programas, por meio de suas ações cotidianas e de suas redes de relacionamento, conforme também destacado nos estudos de Duarte et al. (2012) e Romani-Dias et al. (2017). Ou seja, o principal motor propulsor da internacionalização são os docentes e estes possuem impacto direto no patamar de excelência nacional e internacional atingido pelos programas estudados.

Outro fato evidenciado na pesquisa é que a internacionalização é vista como naturalmente desenvolvida, não havendo o estabelecimento de estratégias específicas pelas coordenações dos programas para o seu desenvolvimento, nem o estabelecimento de diretrizes por níveis hierárquicos superiores dentro da instituição. No entanto, não se pode excluir o papel da CAPES como indutora de políticas de internacionalização nas instituições e em programas de pós-graduação.

Portanto, as práticas, as práxis e os praticantes se imbricam compondo o strategizing, ou seja, a estratégia (de internacionalização) acontecendo rotineiramente no desenrolar das ações cotidianas dos programas de pós-graduação de excelência estudados. Entende-se que a estratégia de internacionalização é uma consequência da atuação conjunta de todos os participantes (docentes, discentes, coordenação, apoio técnico, órgãos externos, etc.), o que vai ao encontro do que está emergindo na literatura recente denominada de open strategy ou estratégia aberta (Nketia, 2016).

Cabe destacar, no entanto, a possibilidade de mudanças no papel dos atores estratégicos da internacionalização, como os docentes, o coordenador dos programas de pós-graduação e os demais setores da instituição envolvidos, diante das características do recém-instituído Programa CAPESPrInt. Considerando o fato de que o programa busca promover um vínculo direto entre a estratégica institucional de internacionalização e a internacionalização dos programas de pós-graduação contemplados pelo edital, a autonomia no papel dos docentes no processo estratégico de internacionalização poderá sofrer influências. Todavia, uma vez que cada instituição contemplada 
Práticas Estratégicas de Internacionalização de Programas de Pós-Graduação: Estudo de Caso em uma Universidade Pública do Sul do Brasil

pelo programa teve autonomia na construção do Projeto Institucional de Internacionalização submetido à CAPES, o papel estratégico desempenhado pelos docentes dos programas participantes poderá apresentar características distintas entre as diferentes instituições contempladas.

A principal contribuição deste estudo está no avanço das discussões acerca da perspectiva da estratégia como prática social que coloca o strategizing (fazer estratégia) como algo que a organização desenvolve no seu cotidiano para atingir os resultados almejados, no caso, relacionando com o que acontece nos programas de pós-graduação (Whittington, 2006; Jarzabkowski et al., 2007). Além disso, evidenciou-se o caráter orgânico atribuído ao desenvolvimento da internacionalização dos programas de pós-graduação e destacou-se os atores desse processo.

A limitação da pesquisa está relacionada à questão do tempo disponível para a coleta dos dados, que não possibilitou, nesta etapa, a realização de entrevistas com docentes e discentes dos programas e outros gestores institucionais além do coordenador, bem como a análise de outros documentos, como os currículos lattes dos docentes e discentes, o que poderia ter contribuído para um estudo mais aprofundado sobre as práxis adotadas e sobre os caminhos percorridos para o atingimento do

\section{REFERÊNCIAS}

Altbach, P. G. (2006). Globalization and the university: Realities in an unequal world. In J. F. Forest, \& P. G. Altbach (Eds.), International Handbook of Higher Education: Part One (Cap. 8, pp. 121-140). Dordrecht: Springer.

Altbach, P. G., Reisberg, L., \& Rumbley, L. E. (2009). Trends in global higher education: Tracking an academic revolution. Paris: UNESCO. Recuperado de http://unesdoc.unesco.org/images/0018/001831/183168 e.pdf

Andrews, K. R. (1971). The Concept of Corporate Strategy. Homewood: Dow JonesIrwin.

Ansoff, H. I. (1965). Corporate Strategy. New York: McGraw-Hill.

Bendelier, S., Kondakci, Y., \& Zawacki-Richter, O. (2018). Two decades of research into the internationalization of higher education: Major themes in the Journal of Studies in International Education (19972016). Journal of Studies in International Education, 22(2), 108-135. Recuperado patamar de excelência esperado pela CAPES. Reforçase aqui que a pesquisa tratou da análise das estratégias no nível dos programas, sob a ótica dos coordenadores, não tendo abordado possíveis estratégias em nível institucional que possam ter colaborado e/ou impactado na internacionalização dos programas estudados, mesmo que indiretamente.

Sugere-se que o escopo desta pesquisa seja ampliado com a inclusão, além dos demais praticantes, de programas de pós-graduação com nota 6 junto à CAPES, os quais também possuem seu grau de internacionalização bem desenvolvido, assim como outras instituições de ensino superior, a fim de comparar possíveis diferenças de acordo com o tipo de instituição analisada (pública/privada). Ainda, sugere-se o estudo com programas conceito 5, para verificar as estratégias e as práticas internacionalização adotadas, buscando identificar a trajetória possível para o desenvolvimento desses programas na busca da excelência.

Para concluir, destaca-se a relevância dos estudos referentes à estratégia de internacionalização dos programas de pós-graduação, colocando-se luz nos estudos sobre open strategy, explicando a influência dos atores organizacionais (tanto gerenciais quanto não gerenciais) e sua participação e envolvimento que geram o compromisso com a estratégia.

http://journals.sagepub.com/doi/pdf/10.1177/10283153 17710093. doi: 10.1177/1028315317710093

Brandt, J. Z., Lavarda, R. A. B., \& Lozano, M.-A. S. L. (2017). Estratégia-como-prática social para a construção da perspectiva de gênero nas políticas públicas em Florianópolis. Revista de Administração Pública, 51(1), 6487. Recuperado de: http://www.scielo.br/pdf/rap/v51n1/0034-7612-rap-5101-00064.pdf. doi: 10.1590/0034-7612147905

Canhada, D. I. D., \& Rese, N. (2009). Contribuições da "estratégia como prática" ao pensamento em estratégia. Revista Brasileira de Estratégia, 2(3), 273-289. Recuperado de https://periodicos.pucpr.br/index.php/REBRAE/article/vie $\underline{w / 13475 / 12893}$

Cardoso, F., \& Lavarda, R. A. B. (2015). Perspectiva da estratégia-como-prática e o processo de formação da estratégia articulada pela média gerência. REAd - Revista Eletrônica de Administração, 21(3), 719-749. Recuperado de http://www.scielo.br/pdf/read/v21n3/1413-2311read-21-03-00719.pdf 
Chandler, A. D. (1962). Strategy and Structure: chapters in the history of the American industrial enterprise. Cambridge: MIT Press.

Coordenação de Aperfeiçoamento de Pessoal de Nível Superior. (2010). Plano Nacional de Pós-Graduação: PNPG 2011-2020 (Vols. 1-2). Brasília: Autor. Recuperado de http://www.capes.gov.br/plano-nacional-de-posgraduacao

Coordenação de Aperfeiçoamento de Pessoal de Nível Superior. (2013a, dezembro 2). Ficha de Avaliação do Programa de Pós-Graduação em Farmacologia da UFSC. Recuperado de http://conteudoweb.capes.gov.br/conteudoweb/Visualiz adorServlet?nome=41001010/008/2013 $008 \quad 41001010$ 024P6 Ficha.pdf\&aplicacao=avaliacaotrienal\&idEtapa $=2$ $\underline{\& a n o=2013 \& \text { tipo=divulga }}$

Coordenação de Aperfeiçoamento de Pessoal de Nível Superior. (2013b, dezembro 19). Ficha de Avaliação do Programa de Pós-Graduação em Química da UFSC. Recuperado de http://conteudoweb.capes.gov.br/conteudoweb/Visualiz adorServlet?nome $=41001010 / 004 / 2013004 \quad 41001010$ 022P3 Ficha.pdf\&aplicacao=avaliacaotrienal\&idEtapa $=2$ \&ano=2013\&tipo=divulga

Coordenação de Aperfeiçoamento de Pessoal de Nível Superior. (2014, abril 16). Ficha de Avaliação do Programa de Pós-Graduação em Engenharia Mecânica da UFSC. Recuperado de http://conteudoweb.capes.gov.br/conteudoweb/Visualiz adorServlet?nome $=41001010 / 013 / 2013 \quad 013 \quad 41001010$ 006P8 Ficha.pdf\&aplicacao=avaliacaotrienal\&idEtapa $=2$ $\underline{\text { \&ano=2013\&tipo=divulga }}$

Coordenação de Aperfeiçoamento de Pessoal de Nível Superior. (2015, junho 22). Sobre avaliação de cursos. Recuperado

de

http://www.capes.gov.br/acessoainformacao/perguntasfrequentes/avaliacao-da-pos-graduacao/7421-sobreavaliacao-de-cursos

Coordenação de Aperfeiçoamento de Pessoal de Nível Superior. (2017a, agosto 7) Primeira Avaliação Quadrienal da CAPES começa em 3 de julho. Recuperado de http://www.capes.gov.br/sala-deimprensa/noticias/8450-primeira-avaliacao-quadrienalda-capes-comeca-em-3-de-julho

Coordenação de Aperfeiçoamento de Pessoal de Nível Superior. (2017b, outubro 31). A internacionalização na Universidade Brasileira: resultados do questionário aplicado pela CAPES. Brasília: CAPES. Recuperado de http://www.capes.gov.br/images/stories/download/diver sos/A-internacionalizacao-nas-IES-brasileiras.pdf

Coordenação de Aperfeiçoamento de Pessoal de Nível Superior. (2017c, novembro 10). Programa Institucional de Internacionalização - Capes-PrInt. Edital no 41/2017.
Recuperado

de

http://www.capes.gov.br/images/stories/download/edita is/10112017-Edital-41-2017-Internacionalizacao-PrInt2.pdf

Coordenação de Aperfeiçoamento de Pessoal de Nível Superior. (2017d, novembro 17). Sobre a avaliação. Recuperado http://www.capes.gov.br/avaliacao/sobre-a-avaliacao

Cunningham, J., \& Harney, B. (2012). Strategy and Strategists. Oxford: Oxford University Press.

De Wit, H. (2013). Internationalisation of higher education, an introduction on the why, how and what. In H. De Wit (Ed.). An Introduction to Higher Education Internationalisation. (pp. 13-46). Milão: Vita e Pensiero.

De Wit, H., \& Hunter, F. (2015). The Future of Internationalization of Higher Education in Europe. International Higher Education, 83, 2-3. Recuperado de https://ejournals.bc.edu/ojs/index.php/ihe/article/view/9 $\underline{073 / 8180 .}$.

doi: http://dx.doi.org/10.6017/ihe.2015.83.9073.

Duarte, R. G., Castro, J. M., Cruz, A. L. A., \& Miura, I. K. (2012). O papel dos relacionamentos interpessoais na internacionalização de instituições de ensino superior. Educação em Revista, 28(1), 343-370. Recuperado de http://www.scielo.br/pdf/edur/v28n1/a15v28n1.pdf

Eisenhardt, K. M. (1989). Building theories from case study research. Academy of management review, 14(4), 532-550.

Godoi, C. K., Bandeira-De-Mello, R., \& Silva, A. B. (Orgs.). (2006). Pesquisa qualitativa em estudos organizacionais: paradigmas, estratégias e métodos. São Paulo: Saraiva.

Goles, T., \& Hirschheim, R. (2000). The paradigm is dead, the paradigm is dead... long live the paradigm: the legacy of Burrell and Morgan. Omega - The International Journal of Management Science, 28(3), p. 249-268. Recuperado de https://www.sciencedirect.com/science/article/pii/S0305 048399000420. doi: https://doi.org/10.1016/S03050483(99)00042-0.

Golsorkhi, D., Rouleau, L., Seidl, D., \& Vaara, E. (2015). Introduction: what is strategy as practice? In D. Golsorkhi, L. Rouleau, D. Seidl, \& E. Vaara. (Eds.), Cambrigde handbook of strategy as practice (2a ed., pp. 1-29). Cambrigde: Cambrigde University Press.

Hudzik, J. K. (2011). Comprehensive internationalization: From concept to action. Washington: NAFSA. Recuperado de https://www.nafsa.org/uploadedFiles/NAFSA Home/Res ource Library Assets/Publications Library/2011 Compre hen Internationalization.pdf. 
Práticas Estratégicas de Internacionalização de Programas de Pós-Graduação: Estudo de Caso em uma Universidade Pública do Sul do Brasil

lasbech, P., \& Lavarda, R. A. B. (2018). Strategy and Practices: A Qualitative Study of a Brazilian Public Healthcare System of Telemedicine. International Journal of Public Sector Management, 31(3), 347-71. Recuperado de

https://www.emeraldinsight.com/doi/pdfplus/10.1108/IJ PSM-12-2016-0207. doi: https://doi.org/10.1108/IJPSM12-2016-0207

Jarzabkowski, P. (2004). Strategy as practice: recursiveness, adaptation, and practices-inuse. Organization studies, 25(4), 529-560. Recuperado de http://journals.sagepub.com/doi/pdf/ 10.1177/0170840604040675.

$\frac{10.1177 / 0170840604040675}{10}$

Jarzabkowski, P. (2005). Strategy as practice: an activity based approach. Londres: Sage Publications.

Jarzabkowski, P., Balogun, J., \& Seidl, D. (2007). Strategizing: the challenges of a practice perspective. Human relations, 60(1), 5-27. Recuperado de http://journals.sagepub.com/doi/pdf/

$\underline{10.1177 / 0018726707075703 .}$.

$\overline{10.1177 / 0018726707075703}$

Jarzabkowski, P., \& Spee, A. (2009). Strategy-aspractice: a review and future directions for the field. International Journal of Management Reviews, 11(1), 69-95. Recuperado de http://onlinelibrary.wiley.com/doi/10.1111/j.1468-

2370.2008.00250.x/pdf. doi: 10.1111/j.14682370.2008.00250.x

Jarzabkowski, P., Kaplan, S., Seidl, D., \& Whittington, R. (2016). On the risk of studying practices in isolation: linking what, who, and how in strategy research. Strategic Organization, 14(3), 248-259. Recuperado de http://journals.sagepub.com/doi/pdf/10.1177/14761270 15604125. doi: 10.1177/1476127015604125

Johnson, G., Melin, L., \& Whittington, R. (2003). Micro strategy and strategizing: towards an activity-based view. Journal of management studies, 40(1), 3-22. Recuperado

http://onlinelibrary.wiley.com/doi/10.1111/1467-

6486.t01-2-00002/epdf. doi: 10.1111/1467-6486.t01-200002

Junquilho, G. S., Almeida, R. A., \& Leite-Da-Silva, A. R. (2012). As "artes do fazer" gestão na escola pública: uma proposta de estudo. Cadernos EBAPE.BR, 10(2), 329-356. Recuperado de http://www.scielo.br/pdf/cebape/v10n2/v10n2a06.pdf. doi: 10.1590/S1679-39512012000200 006

Kerlinger, F. N. (1979). Metodologia da pesquisa em ciências sociais: um tratamento conceitual. São Paulo: EPU.
Knight, J. (2004). Internationalization Remodeled: definition, approaches, and rationales. Journal of Studies in International Education, 8(1), 5-31. Recuperado de http://journals.sagepub.com/doi/10.1177/102831530326 0832.

doi:

https://doi.org/10.1177\%2F1028315303260832.

Knight, J. (2015). International Universities: Misunderstandings and Emerging Models? Journal of Studies in International Education, 19(2), 107-121. Recuperado de https://journals.sagepub.com/doi/pdf/10.1177/1028315 315572899. doi: https://doi.org/10.1177\%2F1028315315572899.

Kuzhabekova, A., Hendel, D. D., \& Chapman, D. W. (2015). Mapping global research on international higher education. Research on Higher Education, 56(8), 861-882. Recuperado

de https://link.springer.com/content/pdf/10.1007\%2Fs1116 2-015-9371-1.pdf. doi: 10.1007/s11162-015-9371-1

Laus, S. P. (2012). A Internacionalização da Educação Superior: um estudo de caso da Universidade Federal de Santa Catarina (Tese de doutorado). Escola de Administração, Universidade Federal da Bahia, Salvador, BA, Brasil.

Laus, S. P., \& Morosini, M. C. (2005). Internationalization of Higher Education in Brazil. In $\mathrm{H}$. De Wit, I. C. Jaramillo, J. Garcel-Ávila, \& J. Knight (Eds.), Higher Education in Latin America: The International Dimension (Cap. 4, pp. 111-148). Washington: The World Bank.

Lavarda, R. A. B., Canet-Giner, M. T., \& Peris-Bonet, F. J. (2010). How middle managers contribute to strategy formation process: connection of strategy processes and strategy practices. Revista de Administração de Empresas, 50(4), 358-370. Recuperado de http://www.scielo.br/pdf/rae/v50n4/02.pdf. doi: 10.1590/S0034-75902010000400002

Leal, F. G., \& Moraes, M. C. B. (2018). Decolonialidade como epistemologia para o campo teórico da internacionalização da Educação Superior. Arquivos Analíticos de Políticas Educativas, 26(87). Recuperado de https://epaa.asu.edu/ojs/article/view/3026/2093. doi: http://dx.doi.org/10.14507/epaa.26.3026.

Lima, M. C. \& Contel, F. B. (2009, maio). Períodos e Motivações da Internacionalização da Educação Superior Brasileira. 5ème Coloque del I'IFBAE, Grenoble, França, 5.

Lima, M. C. \& Contel, F. B. (2011). Internacionalização da educação superior: nações ativas, nações passivas e a geopolítica do conhecimento. São Paulo: Alameda.

Lima, M. C. \& Maranhão, C. M. S. A. (2009). O Sistema de Educação Superior Mundial: entre a internacionalização ativa e passiva. Avaliação, 14(3), 583- 
T. K. Neves, R. A. B. Lavarda \& C. B. Martins

610. Recuperado http://www.scielo.br/pdf/aval/v14n3/a04v14n3.

Maccari, E. A., Lima, M. C., \& Riccio, E. L. (2009). Uso do Sistema de Avaliação da CAPES por programas de pósgraduação em Administração no Brasil. Revista de Ciências da Administração (CAD/UFSC), 11(25), 68-96. Recuperado de

https://periodicos.ufsc.br/index.php/adm/article/view/13 077. doi: https://doi.org/10.5007/21758077.2009v11n25p68

Marrara, T. (2007). Internacionalização da pósgraduação: objetivos, formas e avaliação. Revista Brasileira de Pós-graduação, 4(8), 245-262. Recuperado de

http://ojs.rbpg.capes.gov.br/index.php/rbpg/article/view/ 132/126. doi: 10.21713/2358-2332.2007.v4.132

Minayo, M. C. S. (2002). Ciência, técnica e arte: o desafio da pesquisa social. In M. C. S. Minayo (Org.). Pesquisa social: teoria, método e criatividade (21a ed., Cap. 1, pp. 9-29). Petrópolis: Vozes.

Morgan, G. (1980). Paradigms, metaphors, and puzzle solving in organization theory. Administrative Science Quarterly, 25(4), 605-622.

Morosini, M. C. (2006a). Estado do conhecimento sobre internacionalização da educação superior: conceitos e práticas. Educar em revista, 28, 107-124. Recuperado de http://www.scielo.br/pdf/er/n28/a08n28.pdf. doi: 10.1590/S0104-40602006000200008

Morosini, M. C. (2006b). Internacionalização da Educação Superior: um modelo em construção? In J. L. N. Audy, \& M. C. Morosini (Orgs.). Inovação e Empreendedorismo na Universidade (Cap. 6, pp. 189210). Porto Alegre: Edipucrs.

Morosini, M. C., \& Do Nascimento, L. M. (2017). Internacionalização da Educação Superior no Brasil: a produção recente em teses e dissertações. Educação em Revista, 33, 1-27. Recuperado de http://www.scielo.br/pdf/edur/v33/1982-6621-edur-33e155071.pdf. doi: 10.1590/0102-4698155071

Nketia, B. A. (2016). The influence of open strategizing on organizational members' commitment to strategy. Procedia - Social and Behavioral Sciences, 235, 473-483. Recuperado http://www.sciencedirect.com/science/article/pii/S18770 42816315920. doi: 10.1016/j.sbspro. 2016.11.058

Ramos, M. Y. (2018). Internacionalização da pósgraduação no Brasil: lógica e mecanismos. Educação e Pesquisa, 44 Recuperado de http://www.scielo.br/pdf/ep/v44/1517-9702-ep-S15179702201706161579.pdf. doi: http://dx.doi.org/10.1590/s1517-9702201706161579.
Romani-Dias, M., Carneiro, J. M. T., \& Barbosa, A. S. (2017, outubro). A internacionalização de Instituições de Ensino Superior: proposição de modelo conceitual a partir do papel dos pesquisadores. Encontro da ANPAD EnANPAD 2017, São Paulo, SP, Brasil, 41.

Sampaio, H., \& Saes, P. (2014). Internationalization of higher education: A balance of the literature in Brazil. In $\mathrm{S}$. D. Aupetit, \& V. J. Escobar (Coords.), Internacionalizatión de la educación superior y la ciencia em América Latina: Un estado del arte (pp. 49-75). Caracas: UNESCO-IESALC. Recuperado de http://www.iesalc.unesco.org.ve/dmdocuments/observat orios/movilidad/internacionalizacon_educacion_superior. pdf

Sanderson, G. (2008). A foundation for the internationalization of the academic self. Journal of Studies in International Education, 12(3), 276-307. Recuperado de http://journals.sagepub.com/doi/pdf/10.1177/10283153 07299420. doi: 10.1177/1028315307299420

Seeber, M., Cattaneo, M., Huisman, J., \& Paleari, S. (2016). Why do higher education institutions internationalize? An investigation of the multilevel determinants of internationalization rationales. Higher Education, 72(5), 685-702. Recuperado de https://link.springer.com/content/pdf/10.1007\%2Fs1073 4-015-9971-x.pdf. doi: 10.1007/s10734-015-9971-x

Seeber, M., Meoli, M., \& Cattaneo, M. (2018). How do European higher education institutions internationalize? Studies in Higher Education. doi: https://doi.org/10.1080/03075079.2018.1541449

Silva, A. R. L., Carrieri, A. P., \& Junquilho, G. S. (2011). A estratégia como prática social nas organizações: articulações entre representações sociais, estratégias e táticas cotidianas. Revista de Administração da USP, 46(2), 122-134. Recuperado de https://www.revistas.usp.br/rausp/article/download/445 30/48150. doi: 10.570/rausp1002

Shahjahan, R. A. (2016). International organizations (IOs), epistemic tools of influence, and the colonial geopolitics of knowledge production in higher education policy. Journal of Education Policy, 31(6), 694-710. Recuperado de https://www.tandfonline.com/doi/pdf/10.1080/0268093 9.2016.1206623? needAccess=true. https://doi.org/10.1080/02680939.2016.1206623.

Stake, R. E. (2003). Case studies. In N. K. Denzin, \& Y. S. Lincoln (Eds.). Strategies of qualitative inquiry (2a ed, Cap. 5). London: Sage Publications.

Stallivieri, L. (2017a). Internacionalização e Intercâmbio: dimensões e perspectivas. Curitiba: Appris.

Stallivieri, L. (2017b). Compreendendo a internacionalização da educação superior. Revista de 
Práticas Estratégicas de Internacionalização de Programas de Pós-Graduação: Estudo de Caso em uma Universidade Pública do Sul do Brasil

Educação do COGEIME, 26(50), 15-36. Recuperado de https://www.redemetodista.edu.br/revistas/revistascogeime/index.php/COGEIME/article/view/729/648. doi: http://dx.doi.org/10.15599/0104-

4834/cogeime.v26n50p15-36.

Stein, S. (2017). Internationalization for an uncertain future: tensions, paradoxes, and possibilities. The Review of Higher Education, 41(1), 3-32. Recuperado de https://muse.jhu.edu/article/668564. doi: http://doi.org/10.1353/rhe.2017.0031.

Trochim, W. M. K. (1989). Outcome pattern matching and program theory. Evaluation and program planning, 12(4), 355-366. Recuperado de https://www.sciencedirect.com/science/article/pii/01497 $\underline{18989900529}$

Universidade Federal de Santa Catarina. (2017). UFSC em números 2017. Florianópolis: Autor. Recuperado de http://dpgi.seplan.ufsc.br/files/2013/12/UFSC-EMN\%C3\%9AMEROS-2008-2017.pdf

Vaara, E. \& Whittington, R. (2012). Strategy-aspractice: Taking social practices seriously. The academy of Management Annals, 6(1), 285-336. doi: 10.1080/19416520.2012.672039

Vergara, S. C. (2010). Métodos de pesquisa em administração (4a ed.). São Paulo: Atlas.
Volberda, H. W. (2004). Crise em estratégia: fragmentação, integração ou síntese. RAE - Revista de Administração de Empresas, 44(4), 32-43. Recuperado de https://rae.fgv.br/sites/rae.fgv.br/files/artigos/10.1590 S 0034-75902004000400005.pdf.

Whittington, R. (1996). Strategy as practice. Long range planning, 29(5), 731-735.

Whittington, R. (2002). O que é estratégia. São Paulo: Pioneira Thomson Learning.

Whittington, R. (2006). Completing the practice turn in strategy research. Organization studies, 27(5), 613-634. Recuperado de http://journals.sagepub.com/doi/10.1177/017084060606 4101. doi: 10.1177/0170840606064101

Yin, R. K. (2005). Estudo de caso: planejamento e métodos (3a ed.). Porto Alegre: Bookman.

Zwick, E., Silva, I. C., \& Brito, M. J. (2014). Estratégia como prática social e teoria da ação comunicativa: possíveis aproximações teóricas. Cadernos EBAPE.BR, 12, 384-400. Recuperado de http://www.scielo.br/pdf/cebape/v12nspe/02.pdf. doi: 10.1590/1679-39518694

\section{SOBRE OS AUTORES}

- $\quad$ Thayse Kiatkoski Neves - Universidade Federal de Santa Catarina - UFSC, Santa Catarina, (Brasil). E-mail: thayse.neves@ufsc.br Orcid id: https://orcid.org/0000-0002-7738-5426

- $\quad$ Rosalia Aldraci Barbosa Lavarda - Universidade Federal de Santa Catarina - UFSC, Santa Catarina, (Brasil). Email: rosalia.lavarda@ufsc.br Orcid id: http://orcid.org/0000-0002-1093-4486

- Cibele Barsalini Martins - Universidade Federal de Santa Catarina - UFSC, Santa Catarina, (Brasil). E-mail: cibele.martins@ufsc.br Orcid id: http://orcid.org/0000-0002-7396-0630 
T. K. Neves, R. A. B. Lavarda \& C. B. Martins

\title{
STRATEGIC PRACTICES OF INTERNATIONALIZATION OF GRADUATE PROGRAMS: CASE STUDY IN A PUBLIC UNIVERSITY OF THE SOUTH OF BRAZIL
}

\author{
Thayse Kiatkoski Neves, Rosalia Aldraci Barbosa Lavarda, Cibele Barsalini Martins \\ Federal University of Santa Catarina - UFSC, Santa Catarina, (Brasil)
}

\begin{tabular}{l}
\hline ARTICLE DETAILS \\
\hline Article history: \\
Received: 04 July 2018 \\
Accepted: 19 December 2018 \\
Available online March: 26 th 2019 \\
Double Blind Review System \\
Scientific Editor \\
Ilan Avrichir \\
\hline
\end{tabular}

\section{Keywords}

Strategy as social practice

Internationalization

Graduate programs

\begin{abstract}
The strategy as social practice perspective has emerged as an alternative to the traditional research in the field of organizational strategy. In this perspective, strategy is understood as something people do in their everyday life, and not as something organizations have. The internationalization of Brazilian graduate studies is one of the main goals of the national graduate system. In this respect, the perspective of strategy as social practice can be employed to understand the microdynamics related to the internationalization strategies of graduate programs, to understand how internationalization activities are effectively implemented, and who the actors responsible for implementing them are. This study sought to understand how internationalization strategies of stricto sensu graduate programs of academic excellence, which have obtained the maximum score in CAPES 2013 Triennial Evaluation, of a federal public university are developed in everyday practice. A qualitative methodology research was carried out based on a case study conducted among programs of a public university of the south of Brazil. The results highlighted the distinctive role played by the professors in the internationalization process of graduate programs, and they showed that strategy happens routinely from the day-to-day activities of the programs of excellence studied.
\end{abstract}

(C) 2018 Internext | ESPM. All rights reserved!

Para citar este artigo:

Neves, T., Lavarda, R., \& Martins, C. (2019). Práticas Estratégicas de Internacionalização de Programas de Pós-Graduação: Estudo de Caso em uma Universidade Pública do Sul do Brasil. Revista Eletrônica de Negócios Internacionais, 14(2), 93-110. doi:http://dx.doi.org/10.18568/internext.v14i2.465 